\title{
Importance of timing of post-contrast MRI in rheumatoid arthritis: what happens during the first 60 minutes after IV gadolinium-DTPA?
}

\author{
M Østergaard, M Klarlund
}

\begin{abstract}
Background-Volumes of inflamed synovial membrane determined by magnetic resonance imaging (MRI) are closely related to histopathological synovitis and may predict erosive progression in rheumatoid arthritis (RA). However, after IV injection, leakage of MRI contrast from the synovium gradually compromises the differentiation of synovium from joint fluid.
\end{abstract}

Objective-To determine the time period after IV MRI contrast (gadolinium-DTPA (Gd)) injection in which synovial membrane volume determination is reliable.

Methods-MRI of five RA knees with clinical synovitis was carried out, with axial, $T_{1}$ weighted, spin echo images before IV Gd injection and every 1.75 minutes for 60 minutes post-Gd. By a semiautomated "signal enhancement threshold" method, including voxels with $>35 \%$ or $>45 \%$ relative post-Gd enhancement, synovial membrane volumes were estimated at each time point. At 4.25 minutes post-Gd, volumes were also determined by a more accurate but time consuming "manual method".

Results-The initially observed synoviumeffusion borderline remained clearly visible, and on the same location, within at least the initial 11 minutes post-Gd (that is, within the normal time frame of post-Gd imaging in RA) but started blurring and moving centripetally thereafter. Compared with volumes at all other time points, synovial membrane volumes at 0.75 and 2.50 minutes post-Gd were significantly lower (Wilcoxon-Pratt), suggesting that some synovial membrane areas had not yet exceeded the enhancement threshold. Thereafter, the measured volumes remained practically unchanged.

Conclusion-This study suggests that MR image acquisition in arthritic knee joints should be performed within the initial approximately 10 minutes after gadolinium contrast injection to achieve the most accurate distinction between synovium and joint fluid but that small time variations are not of major importance to the measured synovial membrane volumes.

(Ann Rheum Dis 2001;60:1050-1054)

Contrast enhanced magnetic resonance imaging (MRI) allows assessment of inflammatory and destructive disease manifestations in rheumatoid arthritis (RA). ${ }^{1}$ Volumes of inflamed synovial membrane can be estimated by MRI. ${ }^{2}{ }^{3}$ It has recently been shown that synovial membrane volumes determined by MRI are closely related to histopathological signs of synovitis, ${ }^{4}$ and preliminary data indicate a prognostic value for progressive joint destruction. ${ }^{5}$ Various volume quantification methods have been introduced. A semiautomatic "enhancement threshold" method, based on computerised counting of voxels with a certain post-gadolinium contrast $(\mathrm{Gd})$ signal intensity increase, has been used by several groups. $^{3}$ 6-8

The length of time after contrast injection during which reliable assessment of synovitis can be achieved has been debated. The background for this is that leakage of contrast from the synovial membrane gradually leads to enhancement of the joint fluid and thus may compromise the differentiation of synovium and joint fluid..$^{9-11}$

Using repetitive MRI every 1.75 minutes during one hour after intravenous gadoliniumDTPA (Gd) we aimed at studying the gradual joint fluid enhancement and investigating the resulting effect on synovial membrane volumes measured by the "enhancement threshold" method so that the importance of the exact timing of post-Gd MRI could be assessed.

\section{Patients and methods}

PATIENTS

The study group comprised five patients (four female, one male) with $\mathrm{RA}^{12}$ and clinical signs of knee joint synovitis - that is, joint swelling and tenderness. Patients had a median age of 65 years (range 43-81), disease duration of nine years (5-28), duration of knee symptoms of nine years (4-12), and serum $C$ reactive protein $132 \mathrm{nmol} / 1(<95-482)$. Two patients were receiving slow acting antirheumatic drugs (one methotrexate, one auranofin), three patients low dose oral prednisolone (median 5 $\mathrm{mg}$ /day, range 2.5-10), and three patients nonsteroidal antirheumatic drugs. Intra-articular steroids had not been given within the past three months and no treatment changes had occurred within the past one month

MAGNETIC RESONANCE IMAGING

A 1.0 Tesla Siemens Impact MR unit (Erlangen, Germany) with a dedicated knee coil was used. Sagittal and axial , $\mathrm{T}_{1}$ weighted, spin echo MR images were obtained. At the same time as $0.05 \mathrm{mmol}$ Gd-DTPA (Schering, 
Berlin, Germany)/kg body weight was injected intravenously, the axial sequence was restarted and repeated every 1.75 minutes for 60 minutes.

The parameters of the axial $T_{1}$ weighted sequence were: repetition time/echo time/slice thickness/number of acquisitions/field of view/ matrix size/pixel size $=750 \mathrm{~ms} / 15 \mathrm{~ms} / 5 \mathrm{~mm} / 1 /$ $180 \mathrm{~mm}$ (rectangular) $/ 200 \times 256 / 0.74 \times 0.74$ $\mathrm{mm}$. At each time point the synovial membrane volume was determined twice by the "enhancement threshold" method, with enhancement thresholds of $>35 \%$ and $>45 \%$, respectively (see below).
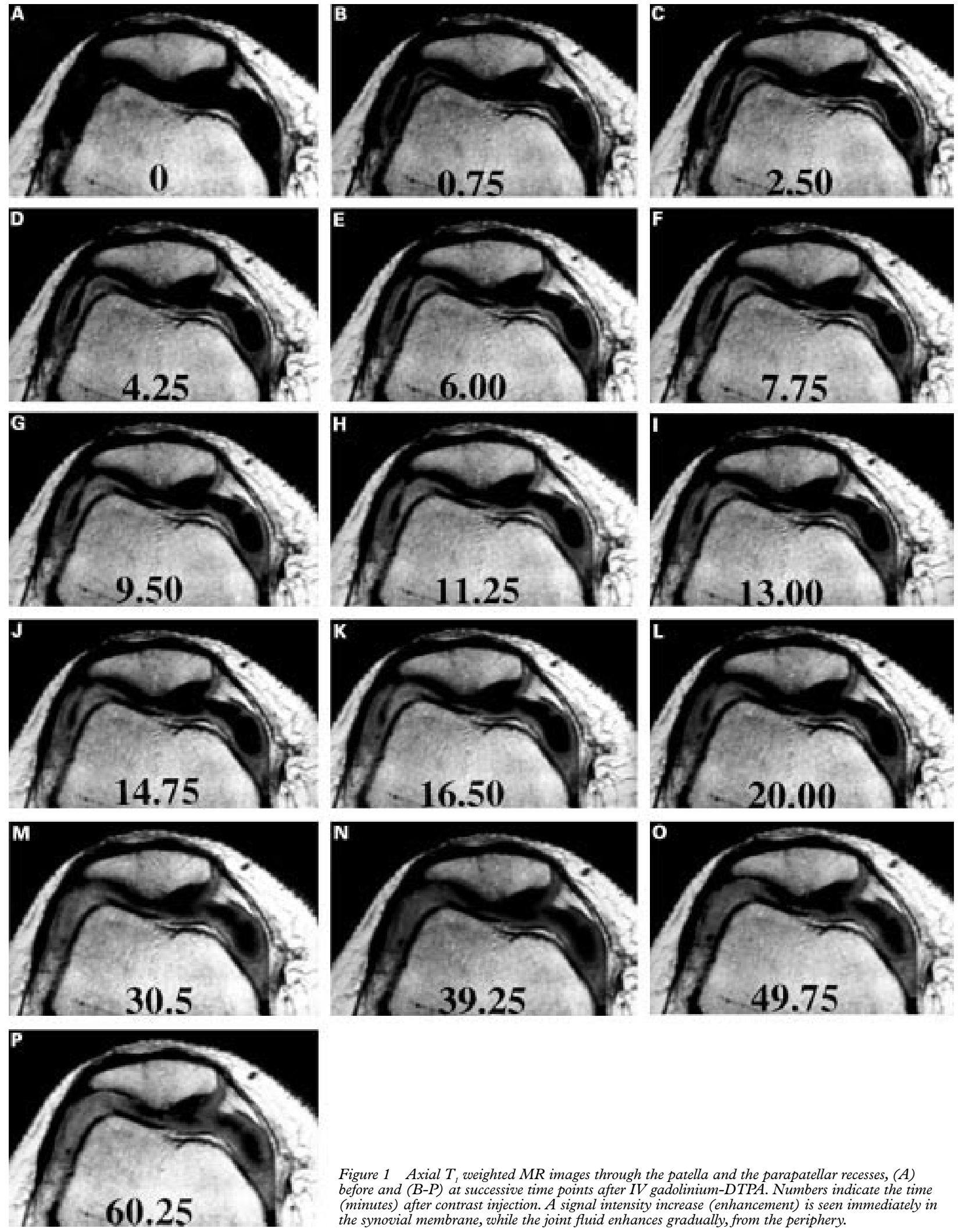

Figure 1 Axial $T_{1}$ weighted MR images through the patella and the parapatellar recesses, $(A)$ before and $(B-P)$ at successive time points after IV gadolinium-DTPA. Numbers indicate the time (minutes) after contrast injection. A signal intensity increase (enhancement) is seen immediately in the synovial membrane, while the joint fluid enhances gradually, from the periphery.

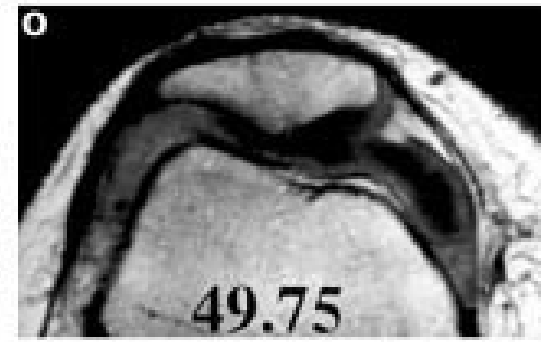



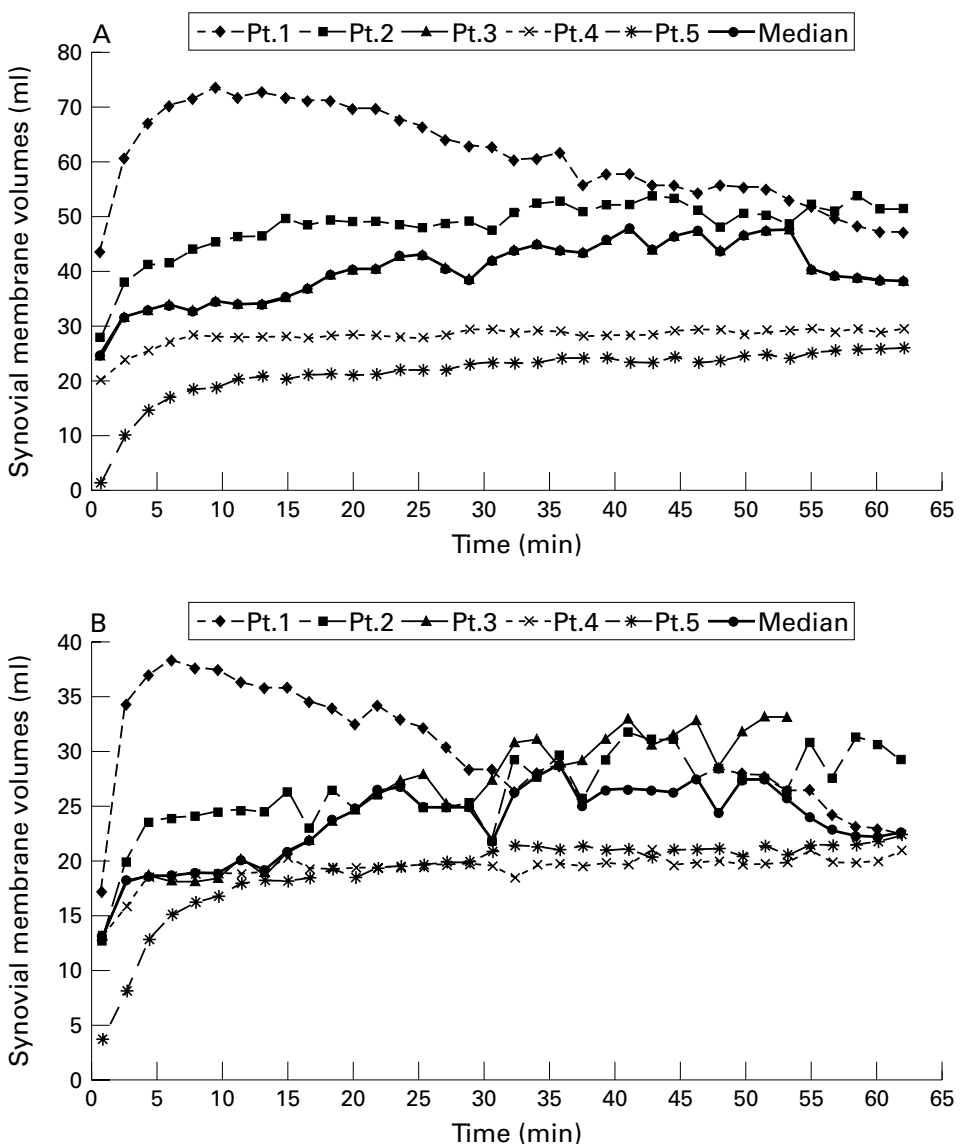

Figure 2 The course of the synovial membrane volume, as estimated by the enhancement threshold method, within the first 60 minutes after IV gadolinium-DTPA. (A)

Enhancement threshold $>35 \%$; (B) enhancement threshold $>45 \%$.

The "post-Gd times" assigned to the individual repetitions were the midpoint of each 1.75 minute repetition. With a $\mathrm{Gd}$ injection time of approximately 15 seconds, the first repetition - that is, the one recorded during $\mathrm{Gd}$ injection, is considered obtained at 45 seconds (0.75 minutes) post-Gd, the following at 2.50 minutes, 4.25 minutes, etc.

DETERMINATION OF SYNOVIAL MEMBRANE VOLUMES BY THE "ENHANCEMENT THRESHOLD" METHOD

By means of the in-house image processing software package XPrime, installed on a Sun Sparc 10 computer (Unix), a rough manual outlining of the areas including synovial tissue was performed on axial images. Extra-articular enhancing tissues, mostly vessels, were excluded. Secondly, a segmentation algorithm was applied, which showed and counted voxels (image points) fulfilling the following criteria: (a) a relative post-Gd signal intensity increase $>45 \%\left(\operatorname{vol}_{45 \%}\right)$ or $>35 \%\left(\operatorname{vol}_{35 \%}\right) ;(b)$ a post-Gd absolute synovial signal intensity $>300$ (corresponding approximately to the mean pre-Gd synovial membrane signal intensity minus 2SD). This criterion was included to avoid noise from low intensity voxels.

Finally, volumes were calculated by multiplication by the voxel size. Methodological details have been reported previously. ${ }^{6}$
DETERMINATION OF SYNOVIAL MEMBRANE VOLUMES BY THE "MANUAL OUTLINING" METHOD From the image set recorded 4.25 minutes post-Gd, the volume was also measured by manual computer assisted synovial membrane outlining. By means of the image processing software, the synovial membrane and the joint fluid of each transverse MRI slice were outlined manually using a computer mouse, and the areas were automatically calculated. Total volumes of synovial membrane and joint fluid were calculated by summation of slices. Methodological details have been described previously. $^{24}$

\section{Results}

Before contrast injection, the synovial membrane and the joint fluid both had an intermediate signal intensity on the $T_{1}$ weighted spin echo images. Visual analysis of post-Gd image sets revealed immediate, marked enhancement of a peripheral rim (corresponding to the synovium) of the joint compartment, followed by gradual enhancement of the adjacent, more centrally located voxels (corresponding to the peripheral parts of the joint fluid). Subsequently, the enhancement approached central joint fluid areas (fig 1).

The borderline between the peripheral rim with immediate enhancement and the neighbouring gradually enhancing voxels remained visible, and on the same location, within at least the initial 11 minutes post-Gd-that is, within the normal time frame of post-Gd imaging in RA. After this, the apparent borderline gradually moved centrally, as the peripheral joint fluid enhancement increased (fig 1).

Synovial membrane volumes, determined from the 4.25 minutes post-Gd images by the time consuming manual method, were 22-63 $\mathrm{ml}$ (median $43 \mathrm{ml}$ ). The corresponding joint fluid volumes were $5-40 \mathrm{ml}$ (median $23 \mathrm{ml}$ ).

Synovial membrane volumes, as measured by the $>35 \%$ enhancement threshold method at the same time (4.25 minutes post-Gd), ranged from 14 to $67 \mathrm{ml}$ (median $33 \mathrm{ml}$ ). These volumes were not significantly different from the manually determined volumes (Wilcoxon-Pratt test, NS). Volumes at 0.75 and 2.50 minutes post-Gd were lower than volumes at 4.25 minutes and later, while volumes thereafter remained almost constant when all five patients were considered together. Figure 2A illustrates the volumes of the individual patients as a function of post-Gd time.

When the higher enhancement threshold of $>45 \%$ was used (fig $2 \mathrm{~B}$ ), the resulting volumes were markedly lower (range 13-37 ml, median $19 \mathrm{ml}$, at 4.25 minutes after $\mathrm{Gd}$ ). However, there were still no statistically significant variations in the measured volumes from 4.25 to 60 minutes post-Gd.

\section{Discussion}

Contrast enhanced magnetic resonance imaging (MRI) allows estimation of volumes of inflamed synovial membrane. ${ }^{2}{ }^{3}$ These MRI determined volumes have been shown to be closely related to histopathological signs of 
synovitis ${ }^{4}$ and may give detailed information on the inflammatory processes in rheumatoid joints. ${ }^{511}{ }^{13-18}$ Furthermore, preliminary data indicate a prognostic value for progressive joint destruction. ${ }^{5}$

To determine the synovial membrane volume, it must be possible to differentiate the synovial membrane from its surroundings. The main problem is the distinction from joint fluid, ${ }^{9-11}{ }^{19}$ because effusions, like the synovium, show post-Gd enhancement on $T_{1}$ weighted images. The enhancement of other adjacent structures, such as cartilage, bone and fatty tissue, is minimal..$^{21}$

As the synovium has no tight junctions or basement membrane, it is permeable to Gd. Consequently, $\mathrm{Gd}$ will diffuse into the joint space causing increasing enhancement of the joint fluid, starting in the periphery and gradually approaching the central parts. ${ }^{9}{ }^{102-24} \mathrm{How}-$ ever, as Gd is delivered to the synovium by the blood flow, it shows almost instantaneous enhancement, ${ }^{9125-27}$ whereas joint fluid enhancement would be expected to be considerably slower because it is caused by diffusion from the synovium. This perception has been supported by pixel by pixel line profile analysis of the time dependent enhancement at the synovium-fluid border: a distinct change of the shape of the enhancement curve (steep versus flat) between adjacent pixels was found at a certain border, presumably corresponding to the synovium-fluid interface. ${ }^{28}$

By repetitive MRI during 60 minutes after intravenous $\mathrm{Gd}$ injection, we investigated the gradual blurring of the synovium-effusion borderline and its consequence for synovial membrane volume measurements. Five RA knees with clinical and MRI signs of synovitis were studied. We found that a peripheral rim, undoubtedly the synovial membrane, showed instantaneous marked enhancement after IV Gd injections (fig 1). Furthermore, despite incipient enhancement of the adjacent voxels, corresponding to the most peripheral parts of the effusion, this did not hinder the distinction between initially enhanced and initially unenhanced voxels during the initial 11 minutes post-Gd. Thus the phenomenon of joint effusion enhancement does not seem to be quantitatively important to MRI determined knee joint synovial membrane volumes within the normal time frame of post-Gd-DTPA imaging. Later than 11 minutes post-Gd, the initial borderline became gradually indistinguishable (fig 1).

The results of this study are in accordance with previous studies, which indicated that at least in the initial 10 minutes post-contrast, other sources of variation, particularly coincidental variations in the outlining and partial volume artefacts, are more important to the measured synovial membrane volumes than misinterpretation of the synovium-effusion borderline. ${ }^{11} 29$

The fact that a higher threshold ( $>45 \%)$, which resulted in too low absolute volumes, also showed only small changes during the one hour post-contrast follow up period, further supports the view that the enhancement threshold method withstands minor variations in the post-Gd timing.

In a previous study the variation between volumes determined from two sets of MR images obtained $2-5$ days apart by the "enhancement threshold" method (threshold $>40 \%$ ) was $10-15 \%$ (inter-MRI variation). ${ }^{6}$ Reproducibility data of the methods have been reported previously ${ }^{26713}$ and summarised by Østergaard. ${ }^{11}$

In view of the observed increasing joint fluid enhancement occurring after the initial post-Gd quarter, a gradual increase in the measured synovial membrane volumes might have been expected. However, the measured volumes remained almost unchanged during the rest of the one hour observation time. This lack of significant volume changes, despite the gradual joint fluid enhancement, is probably due to the fact that the fluid seldom exceeded the enhancement threshold and, furthermore, that the synovial membrane signal intensity gradually decreased as Gd washed out, causing some "true synovial membrane voxels" to have signal intensities below the enhancement threshold. Similarly, the fact that the volumes measured earlier than 4.75 minutes post-Gd were low, does not indicate that the synovium was not visible at 2.50 minutes post-Gd, but that some synovial membrane voxels did not rapidly exceed the enhancement threshold of $45 \%$ or $35 \%$. These problems are inherent in the "enhancement threshold method" and may be avoided by the more time consuming "manual outlining method". ${ }^{61129}$

This study suggests that MR image acquisition in arthritic knee joints should be performed within the initial 10 minutes post-Gd contrast injection to achieve the most accurate distinction between synovium and joint fluid but that small time variations are not of major importance to the measured volumes.

The authors thank Jens Arnth Jensen and Poul Ring for development of image processing software. We acknowledge the
Foundation of 17-12-81, the University of Copenhagen, the Danish Rheumatism Association, the Thomas and Elisabeth Frølund Nielsen Foundation, the Danish Medical Research Frølund Nielsen Foundation, the Danish Medical Research
Council, Johann and Hanne Weimann born Seedorf's grant, Council, Johann and Hanne Weimann born Seedorf's grant,
Karen Marie Jørgensen and Datters grant and the Danish HosKaren Marie Jørgensen and Datters grant and the Danish Hos-
pital Foundation for Medical Research, Region of Copenhagen, pital Foundation for Medical Research, Region of Copenhagen, The Faroe Islands, and Greenland for financial support. Scher-
ing Diagnostika, Denmark, is acknowledged for providing the ing Diagnostika, Denmark, is acknowledged for providing the
contrast agent.

1 Kursunoglu-Brahme S, Riccio T, Weisman MH, Resnick D, Zvaifler N, Sanders ME, et al. Rheumatoid knee: role of Zvaifler N, Sanders $\mathrm{ME}$, et al. Rheumatoid knee. role of 1990;176:831-5.

2 Østergaard M, Gideon P, Henriksen O, Lorenzen I. Synovial volume - a marker of disease severity in rheumatoid arthritis? Quantification by MRI. Scand J Rheumatol 1994;23: tis? Quan -202 .

3 Waterton JC, Rajanayagam V, Ross BD, Brown D, Whittemore A, Johnstone D. Magnetic resonance methods for measurement of disease progression in rheumatoid arthritis. Magn Reson Imaging 1993;11:1033-8.

4 Østergaard M, Stoltenberg M, Løvgreen-Nielsen P, Volck B, Jensen $\mathrm{CH}$, Lorenzen I. Magnetic resonance imagingdetermined synovial membrane and joint effusion volumes in rheumatoid arthritis and osteoarthritis. Comparison with the macroscopic and microscopic appearance of the synovium. Arthritis Rheum 1997;40:1856-67.

5 Østergaard M, Hansen M, Stoltenberg M, Gideon P, Klarlund $M$, Jensen $\mathrm{KE}$, et al. Magnetic resonance imagingdetermined synovial membrane volume as a marker of disease activity and a predictor of progressive joint destruction in the wrists of patients with rheumatoid arthritis. Arthritis Rheum 1999;42:918-29.

6 Østergaard M. Different approaches to synovial membrane volume determination by magnetic resonance imaging: manual versus automated segmentation. Br J Rheumatol 1997;36:1166-77. 
7 Creamer P, Keen M, Zananiri F, Waterton JC, Maciewicz $\mathrm{RA}$, Oliver $\mathrm{C}$, et al. Quantitative magnetic resonance imaging of the knee: a method of measuring response to intra-articular treatments. Ann Rheum Dis 1997;56:37881.

8 Clunie G, Hall-Craggs MA, Paley MN, King A, Wilkinson ID, Ell PJ, et al. Measurement of synovial lining volume by magnetic resonance imaging of the knee in chronic synovitis. Ann Rheum Dis 1997;56:526-34.

9 Yamato M, Tamai K, Yamaguchi T, Ohno W. MRI of the knee in rheumatoid arthritis: Gd-DTPA perfusion dynamics. J Comput Assist Tomogr 1993;17:781-5.

10 Winalski CS, Palmer WE, Rosenthal DI, Weissman BN. Magnetic resonance imaging of rheumatoid arthritis. Radiol Clin North Am 1996;34:243-58.

11 Østergaard M. Magnetic resonance imaging in rheumatoid arthritis. Quantitative methods for assessment of the inflammatory process in peripheral joints (doctoral thesis). inflammatory process in periphe

12 Arnett FC, Edworthy SM, Bloch DA, McShane DJ, Fries JF, Cooper NS, et al. The American Rheumatism AssociaJF, Cooper NS, et al. The American Rheumatism Associatoid arthritis. Arthritis Rheum 1988;31:315-24.

13 Østergaard M, Stoltenberg M, Gideon P, Sørensen K, Henriksen $\mathrm{O}$, Lorenzen I. Changes in synovial membrane and joint effusion volumes following intraarticular methylprednisolone. Quantitative assessment of inflammatory and destructive changes in rheumatoid arthritis by MRI. J Rheumatol 1996;23:1151-61.

14 Palmer WE, Rosenthal DI, Schoenberg OI, Fischman AJ, Simon LS, Rubin RH, et al. Quantification of inflammation in the wrist with gadolinium-enhanced MR imaging and PET with 2-[F-18]-fluoro-2-deoxy-D-glucose. Radiology 1995; 196:647-55

15 Polisson RP, Schoenberg OI, Fischman A, Rubin R, Simon LS, Rosenthal D, et al. Use of magnetic resonance imaging LS, Rosenthal D, et al. Use of magne in the assessment and positron ef synovial volume and glucose metabolism in patients with

16 Sugimoto H, Takeda A, Kano S. Assessment of disea activity in rheumatoid arthritis using magnetic resonance activity in rheumatoid arthritis using magnetic resonance imaging: quantification of pan

17 Klarlund M. Magnetic resonance imaging of wrist and finger joints in rheumatoid arthritis and early unclassified polyarthritis [PhD thesis]. University of Copenhagen, 2000.

18 Østergaard M, Ejbjerg B, Stoltenberg M, Gideon P, Volck B, Skov K, et al. Quantitative magnetic resonance imaging as marker of synovial membrane regeneration and recurrence of synovitis after arthroscopic knee joint synovectomy - a one year follow up study. Ann Rheum Dis 2001;60:233-6.
19 Peterfy CG, Majumdar S, Lang P, van Dijke CF, Sack K, Genant HK. MR imaging of the arthritic knee: improved discrimination of cartilage, synovium, and effusion with pulsed saturation transfer and fat-suppressed T1-weighted sequences. Radiology 1994;191:413-19.

20 Østergaard M, Court-Payen M, Gideon P, Wieslander S, Cortsen $\mathrm{M}$, Lorenzen I, et al. Ultrasonography in arthritis of the knee. A comparison with MR imaging. Acta Radiol $1995 ; 36: 19-26$

21 Østergaard M, Stoltenberg M, Løvgreen-Nielsen P, Volck B, Sonne-Holm S, Lorenzen I. Quantification of synovitis by MRI: correlation between dynamic and static gadoliniumenhanced MRI and microscopic and macroscopic signs of synovial inflammation. Magn Reson Imaging 1998;16: 753-8.

22 Hervé-Somma CMP, Sebag GH, Prieur A, Bonnerot V, Lallemand DP. Juvenile rheumatoid arthritis of the knee: MR evaluation with Gd-DOTA. Radiology 1992;182:93-8.

23 Drapé J, Thelen P, Gay-Depassier P, Silbermann O, Benacerraf R. Intraarticular diffusion of Gd-DOTA after intravenous injection in the knee: MR imaging evaluation. Radiology 1993;188:227-34.

24 Winalski CS, Aliabadi P, Wright RJ, Shortkroff S, Sledge $\mathrm{CB}$, Weissman BN. Enhancement of joint fluid with intravenously administered gadopentetate dimeglumine: technique, rationale, and implications. Radiology 1993;187: 179-85.

25 Reiser MF, Bongartz GP, Erlemann R, Schneider M, Pauly T, Sittek H, et al. Gadolinium-DTPA in rheumatoid arthritis and related diseases: first results with dynamic magnetic resonance imaging. Skeletal Radiol 1989;18:591-7.

26 König H, Sieper J, Wolf KJ. Rheumatoid arthritis: evaluation of hypervascular and fibrous pannus with dynamic MR imaging enhanced with gd-DTPA. Radiology 1990;176: 473-7.

27 Østergaard M, Stoltenberg M, Henriksen O, Lorenzen I. Quantitative assessment of synovial inflammation by dynamic gadolinium-enhanced MRI. A study of the effect of intraarticular methylprednisolone on the rate of early synovial enhancement. Br J Rheumatol 1996;35:50-9.

28 Gaffney K, Cookson J, Blake D, Coumbe A, Blades S. Quantification of rheumatoid synovitis by magnetic resonance imaging. Arthritis Rheum 1995;38:1610-17.

29 Østergaard M, Stoltenberg M, Henriksen O, Lorenzen I. The accuracy of MRI-determined synovial membrane and oint effusion volumes in arthritis. A comparison of preand post-aspiration volumes. Scand J Rheumatol 1995;24: $305-11$ 